\title{
Optical to X-rays SNe light curves following shock breakout through a thick wind
}

\author{
Gilad Svirski ${ }^{1}$, Ehud Nakar ${ }^{1}$ and Re'em Sari ${ }^{2}$ \\ ${ }^{1}$ Raymond and Beverly Sackler School of Physics \& Astronomy, Tel Aviv University, \\ Tel Aviv 69978, Israel \\ ${ }^{2}$ Racah Institute for Physics, The Hebrew University, Jerusalem 91904, Israel
}

\begin{abstract}
We present luminosity and temperature light curves following supernova shock breakouts through a thick wind. These events are very luminous and their spectrum may contain both an X-ray and a UV/optical component. For breakout pulse durations between a week and a month, the X-ray component luminosity peaks $100-500$ days after the explosion, respectively.
\end{abstract}

\section{A shock breakout through a thick wind}

Supernovae (SNe) shock breakouts through a thick wind last much longer than breakouts from a stellar surface, and are thus easier to detect. If a star is surrounded by a wind with an optical depth $\tau_{w}>c / v$, where $\mathrm{v}$ is the shock speed, then the shock continues into the wind without releasing photons to the observer. Once the wind optical depth drops to $\mathrm{c} / \mathrm{v}$ the shock is breaking out of the wind, releasing all its energy as an intense pulse. Following breakout, the radiation mediated shock is replaced by a collisionless shock. We examine the light curve and the observed spectrum at and following the breakout (see also Chevalier \& Irwin 2012).

\section{Main conclusions}

Wind breakouts are very luminous, $10^{43-44} \mathrm{erg} / \mathrm{s}$ for breakout times of days or longer. Two spectral components are observed: a UV soft component, dominating the luminosity at breakout with temperatures of $10^{4}-10^{6} \mathrm{~K}$, depending on the shock breakout velocity. A hard component (X-rays $/ \gamma$-rays), contributing a fraction of $10^{-4}$ of the soft component luminosity at breakout, rising quickly to domination at $\sim 10-50$ breakout times, with a breakout temperature of $\sim 0.1 \mathrm{keV}$, rising to $1-100 \mathrm{keV}$ at peak. The light curve and spectrum evolution details depend mostly on the breakout time. In terms of prospects for X-rays and soft $\gamma$-rays detection, it is best to observe $100-500$ days after explosions with breakout timescales between a week and a month.

\section{Processes, timescales and light curves}

All the shock internal energy is radiated away (i.e, fast cooling). The bolometric luminosity depends only on the shock velocity and external density, independent of the spectrum. Electrons are heated behind the collisionless shock to $60 \mathrm{keV}$ (Katz et al. 2011). These electrons are the energy source of the emission. The soft component is free-free emission of the unshocked wind, heated by the radiation of the post-shock hot electrons. The hard component photons are free-free emission of the $60 \mathrm{keV}$ post-shock electrons, softened during diffusion through the unshocked wind. The hard component luminosity is suppressed by inverse Compton (IC) cooling of the hot electrons over the 

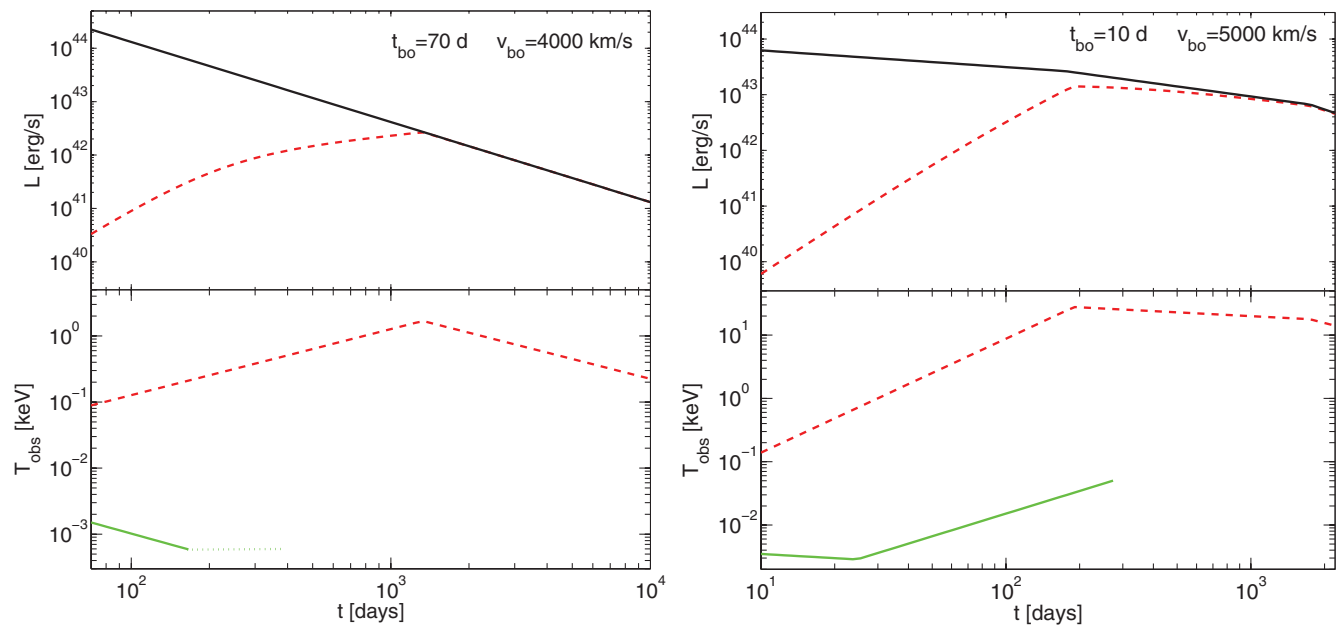

Figure 1. Left: Late breakout $\left(t_{S P}<t_{b o}\right)$. Breakout after the entire ejecta is shocked. Brightest emission, released as optical-UV flash. X-rays observed only long after breakout, if the wind is not terminated by then. Figure parameters chosen to fit SN 2006gy. Solid lines are bolometric luminosity and temperature of the soft component. Dashed lines are the hard component luminosity and temperature. Right: Early breakout $\left(t_{b o}<t_{\text {hard }}<t_{S P}\right)$. Soft component temperature roughly constant while in equilibrium, climbs quickly afterwards. X-rays $/ \gamma$-rays peak luminosity, at 100-300 d after the SN explosion, is similar to breakout bolometric luminosity. A larger $t_{b o}$ yields a brighter X-ray event. May explain SNe like PTF 09uj (Ofek et al. 2010).

soft component and by Compton losses during diffusion. Following breakout, as the wind opacity decreases, both the IC cooling and the Compton loses decrease. As a result the hard component gains dominance over the soft component with time.

Depending on the shock velocity, the breakout radiation may deviate from thermal equilibrium. In such case the observed breakout temperature is high and it depends strongly on the shock velocity. For further discussion see Weaver (1976), Katz et al. (2010) and Nakar \& Sari (2010).

Three timescales are involved: (1) the breakout time, (2) the time when the hard component luminosity becomes dominant, and (3) the transition of the forward shock to a snowplow expansion (when mass of the swept wind becomes comparable to the ejecta mass). The temporal order of these timescales is determined by the breakout time and dictates three evolution scenarios which we denote as early, intermediate and late breakouts. The early and late breakout scenarios are depicted and described in Figure 1. This poster is a summary of Svirski et al. (2012)

\section{References}

Chevalier, R. A. \& Irwin, C. M. 2012, ApJL, 747, L17

Katz, B., Budnik, R., \& Waxman, E. 2010, ApJ, 716, 781

Katz, B., Sapir, N., \& Waxman, E. 2011, ArXiv e-prints

Nakar, E. \& Sari, R. 2010, ApJ, 725, 904

Ofek, E. O., et al. 2010, ApJ, 724, 1396

Svirski, G., Nakar, E., \& Sari, R. 2012, ArXiv e-prints

Weaver, T. A. 1976, ApJS, 32, 233 